\title{
Outcomes Assessment of an International Engineering Experience
}

\author{
David DiBiasio \\ Worcester Polytechnic Institute
}

Study abroad experiences have long been a large part of an undergraduate liberal arts education. However, only recently have engineering students begun to take advantage of the great benefits available from an international experience. This change is partly driven by the increasingly global nature of engineering and by requirement under ABET's EC 2000 accreditation policies. A result of this is an emergence of different models of engineering study abroad such as industrial internships, semester at sea programs, service-oriented models, and traditional exchange programs. A diversity of models means a variety of student experiences and hence different student outcomes. Measuring student outcomes and understanding the learning experience is critical for continuous improvement and satisfying accreditation agencies. In this paper we describe our international program and its relation to ABET-related student outcomes such as multidisciplinary teamwork, understanding engineering in a global society, contemporary issues, life-long learning, and written communication skills. The paper will focus on our assessment process.

\section{Background}

All students at Worcester Polytechnic Institute must complete a multidisciplinary project that examines technology-society interactions. The project is called an Interactive Qualifying Project (IQP). Students research, address and report on a problem examining how science or technology interacts with cultures, societal structures, and values. Project objectives include enabling students to understand, as citizens and as professionals, how their careers will affect the larger society of which they are a part. Projects are done in small, multidisciplinary teams, they are broad and integrative, are not limited to major field, and are equivalent in credit to three courses.

Since the inception of this academic exercise, we have tried to find ways to get students offcampus to do these projects. The first such resident project center was set up in Washington, DC 25 years ago and since then we have expanded the program throughout the world. Now, nearly $2 / 3$ of our students travel internationally to conduct these projects. We send more engineering students abroad than any other US university, and we are second in the nation for doctoral universities for the percent of our total student body that studies abroad. ${ }^{1}$ The details of our program structure and operation are presented elsewhere. ${ }^{2,3}$ Space constraints prevent detailed description of the program and typical student projects. However, shown below is one example of a project completed at our Bangkok center. WPI has developed an administrative division, the Interdisciplinary and Global Studies Division (IGSD) to run this large program, and not only maintain but improve the quality of the students' IQP experience. In this paper, we present our assessment program, and focus on our international projects. 


\section{International IQP Example \\ Sustainability of Farming Systems in Northeast Thailand}

Sponsors: International Board of Soil Resources and Management

and the Ubon Rice Research Center

Students: Electrical Engineer, Mechanical Engineer \&

Biotechnologist

Advisor: Faculty in the Computer Science Department

Students interviewed Thai rice farmers to collect economic and agricultural information relating to farming practices and farm life. A nutrient balance assessment and socioeconomic profile were configured for each farm, and recommendations were developed for the farmers. Results of this project were presented to IBSRAM, the URRC and the Thai Department of Agricultural Extensions.

The International Project Center Structure

The IGSD is charged with administrating all aspects of the IQP program including student selection, site and project development, risk management, and academic quality. To put our assessment efforts in context, we must first outline the overall system structure.

Each international center is residential. Students travel to the site for a 2-month period, with a resident faculty advisor to conduct the projects. Local government, industry, nonprofit organizations, and sometimes universities sponsor projects. Each sponsor provides a liaison responsible for overseeing student teams working with the agency. A WPI faculty member serves as project center director----responsible for setting up projects, general academic issues, and overseeing center operation. A local coordinator, who is a permanent resident at the site, assists the center director particularly with housing and logistical concerns. Student preparation for the experience includes formal coursework taught by WPI faculty, and orientation/cultural preparation taught by WPI professional staff. The same staff handles health and travel issues, risk management, and re-entry issues. At the completion of a project, each student team submits a substantial written report to the sponsoring agency and the faculty advisor. In addition, a formal presentation is made to the agency.

Our goal is to maximize the benefits of this experience not only for the students, but also for all involved. Developing a comprehensive assessment plan for the entire program, given its complex nature, is indeed a challenge. A variety of surveys, interviews and other tools are used to evaluate many aspects of the operation. The main product-oriented evidence currently used to assess student outcomes is the written report. New initiatives are underway in a variety of areas. We focus here on the student reports, and describe some of our results and continuous improvement practices.

\section{The Assessment Process}

The author, Assessment Coordinator for the IGSD, directs the process. The current major evaluation tool for student work is that all reports submitted for grades during a calendar year are read each summer and evaluated by a team of paid faculty reviewers. This practice was 
established several years ago. Although it probes only the product, we have found it quite useful in identifying characteristics of high quality projects.

Each spring a team of reviewers are identified and recruited. They meet for two half-day workshops for training and calibration. We have developed an evaluation form used to guide the assessment of each report. During the first half-day the form is reviewed, discussed, and updated as appropriate. Over the past two years we have added sections related to ABET EC 2000 criteria that we feel the IQP potentially addresses. We have also spent considerable effort writing rubrics to standardize the evaluation. Each reviewer is then given the same three project reports to read and evaluate using the form. We convene for a second half-day to debrief everyone's evaluation, attempt to calibrate each other against the rubrics, and minimize variance in application of the rubrics. Very often rubrics are rewritten on the basis of the discussion.

Each reviewer is then randomly assigned about 20 reports to read and evaluate over the summer. They have a completion deadline by which all evaluation forms must be submitted. The data in the forms is then entered into a database for analysis. Our evaluation form contains questions that cover everything from project objectives, quality of the literature review, application of appropriate methodologies, findings and analysis of data, achievement of IQP goals, quality of the writing and presentation, and several EC 2000 outcomes. These outcomes are ability to function on multidisciplinary teams, life-long learning, impact of engineering in a global and societal context, knowledge of contemporary issues, and understanding professional and ethical responsibility. Copies of the form are available from the author. Most IQP reports approach 100 pages in length and the evaluation form is 10 pages, with 13 questions including many subquestions and comment entries. Hence, the reviewer's task is not a small one!

After all data is entered, the Assessment Coordinator analyzes the results and writes a report to the WPI community. This is in progress as of the writing of this paper. After the report is prepared the Dean of the IGSD works with the Assessment Coordinator on continuous improvement issues. These may involve changes in the student preparation, advisor training, sponsor consultation, resource allocation, or any other issues identified as problematic from the review process.

\section{Example Evaluation Questions, Rubrics, and Results}

We highlight here a few typical results and present data specific to several of the student outcomes mentioned above. Over the past few years we have found that there is a strong, statistically significant correlation between project report quality and high ratings on the literature review, methodology, and achievement of IQP goals questions. Surprisingly, there is also a strong positive correlation between overall report page length and report quality. The message to advisors is that student teams that do high quality background work, apply valid and appropriate methodologies usually produce high quality reports. Since the preparation phase for our international IQP projects is significantly greater than that for on-campus projects, and that preparation heavily emphasizes background and methodologies, it is not surprising that international projects score a full point higher (five-point scale) in an overall quality rating. Part of our improvement efforts is aimed at trying to translate that preparation advantage into our oncampus IQP projects. 
Described below are a few specific examples (primarily related to EC 2000) with our evaluation approach, rubric definition, and preliminary results. In all cases ratings are based upon a 5-point scale.

\section{An Ability to Function on Multidisciplinary Teams}

Measuring students' ability to function on a multidisciplinary presents challenges involving both process and product evaluations. We are working on the process issues, and are attempting to obtain information from the student report. Reviewers are asked to determine "the extent to which the report examines topics using techniques from more than one discipline and/or from disciplines other than the students' majors."

\section{Rating 5: excellent}

\section{Multidisciplinary Team Rubric}

The project makes major and effective use of extensive background knowledge or recognized, respected, and appropriate methodologies drawn from more than one discipline and/ or from disciplines other than the students' majors. (Implies a rating of 4 or higher on methodology and overall quality)

\section{Rating 3: acceptable}

The project makes significant use of background knowledge or appropriate methodologies drawn from more than one discipline and/ or from disciplines other than the students' majors. (Implies a rating of 3 or higher on methodology and overall quality)

\section{Rating 1: poor}

The project makes only peripheral use of background knowledge or methodologies drawn from more than one discipline and/ or from disciplines other than the students' majors.

Average ratings for off-campus projects for this question were 4.1 while the overall average was 3.2. This indicates international projects tend to be more multidisciplinary in nature. Overall, more than $75 \%$ of the IQPs done in 1999 met acceptable or better standards for multidisciplinary teamwork. This indicates that we have some work to do to address the remaining $25 \%$.

\section{A Recognition of the Need to Engage in Life-Long Learning}

This outcome is measured by rating "the extent to which the students acquired and applied knowledge not obtained from prior course work." Preliminary results for this question are not available as of the writing of this paper, but will be presented at the conference. 


\section{Rating 5: excellent}

\section{Life-Long Learning Rubric}

The project contains an extensive, critical review of the literature on a topic and/or makes extensive and effective use of recognized, respected, and appropriate methodologies not likely to have been covered in the students' coursework.

\section{Rating 4: acceptable}

The project contains an acceptable review of the literature on a topic and/or makes significant use of appropriate methodologies not likely to have been covered in the students' coursework.

\section{Rating 1: poor}

The project makes only very limited use of background knowledge or methodologies not likely to have been covered in the students' coursework.

An Ability to Understand the Impact of Engineering Solutions in a Global/Societal Context Since this is a major goal of the IQP it is expected that most, if not all reports, show evidence of this. We measure it not only by evaluating whether the reports show evidence of several of published goals of the IQP, but also by rating students' demonstration of "evaluating the impact of engineering or technological solutions on society." The rubric is shown below.

\section{Rating 5: excellent}

\section{Impact of Engineering Solutions on Society Rubric}

The project is focused heavily, if not entirely, on such an impact and evaluates it effectively using the most appropriate methodologies. (Implies a rating of 4 or higher on methodology and overall quality)

\section{Rating 3: acceptable}

Evaluation of such an impact is a significant component of the project and was conducted using sensible methods (if not state of the art). (Implies a rating of 3 or higher on methodology and overall quality)

\section{Rating 1: poor}

Evaluation of such an impact is a relatively peripheral or incidental component of the project and appropriate methodologies either were not employed or shed little light on this issue.

We also specifically evaluate "the extent of the students' exposure to global/societal issues and/or foreign cultures due to project location and topic." The rubric for this evaluation is below. In 1999, 40\% of the reports represented work done outside of the US and the average rating for these questions was 3.8. Overall $37 \%$ of the reports did not show evidence of "global" issues. These results are consistent with what we expect based upon the numbers of students traveling internationally, but also indicate that for those students who remain on campus for their IQP satisfaction of the EC 2000 "global" outcome is an issue. Furthermore, 32\% of the reports did not show evidence of the social responsibility of engineers. This means we also have a deficit in this area. 


\section{Rating 5: excellent}

\section{Exposure to Global Issues and/or Foreign Cultures Rubric}

The project was conducted at a foreign off-campus site and dealt, in a substantive fashion, with topics that were clearly global in nature or international in scope. If conducted on campus, the project focused on and effectively analyzed topics that were clearly identified as global or international.

\section{Rating 3: acceptable}

The project was conducted at a foreign off-campus site or dealt, in a substantive fashion, with topics that were clearly global in nature or international in scope.

\section{Rating 1: poor}

The project was conducted on campus and contained only oblique indications that the students were aware that some of the problems being addressed were global or international in character.

\section{A Knowledge of Contemporary Issues}

This measurement is still somewhat fuzzy since the term "contemporary issues" is subject to wide interpretation. Currently we evaluate the "extent to which the project report indicated an understanding or appreciation of contemporary issues", using the rubric below.

\section{Rating 5: excellent}

\section{Contemporary Issues Rubric}

The project dealt with an aspect of an issue that is contemporary or aids in understanding contemporary issues. The report, most likely the introduction and/or the literature review, indicated that the students understood clearly the detailed scope and character of the larger societal issue within which their specific topic was imbedded and appreciated its significance.

\section{Rating 3: acceptable}

The project dealt with an aspect of an issue that is contemporary or aids in understanding contemporary issues. The report, most likely the introduction and/or the literature review, indicated that the students understood and appreciated the basic societal issue(s) within which their specific topic was imbedded.

\section{Rating 1: poor}

The project dealt with an aspect of an issue that is contemporary or aids in understanding contemporary issues, but the report says little about the basic societal issue(s) within which the specific topic was imbedded.

Overall only $5.7 \%$ of the reports were absent any evidence of student understanding of contemporary issues. The average rating for international projects was 3.9, indicating that the global project experience produces an acceptable outcome in this category.

\section{An Ability to Communicate Effectively}

For this year's evaluation we made a major change in our evaluation of writing quality. Assisted by the Director of the Center for Communication Across the Curriculum, evaluators are now 
asked to examine the report in four areas. We make no attempt to differentiate individual student contributions since the reports are a collaborative effort. The four areas are:

- understanding of audience (appropriate tone, point of view, use of passive/active voice)

- global and local coherence (appropriate overall structure, report sections complement and advance project objectives; unification of individual paragraphs, appropriate sentence structure and grammar)

- appropriate use of sources (sources integrated into text, critically engaged, properly documented)

- quality of document design (appropriate and clear visuals, proper labeling, visuals and text are complementary)

Since these evaluation questions are new, our rubric (shown below) is relatively crude. Further refinements are planned.

\section{Rating 5: excellent}

\section{Written Communication Rubric}

The rhetorical element enhances the report content.

\section{Rating 3: acceptable}

The rhetorical element is present but neither detracts from nor enhances the report content.

\section{Rating 1: poor}

The particular rhetorical element is absent or so poorly executed that it detracts from the communication of the report content.

Data are not yet available for this expanded evaluation of written communication outcomes, but will be presented at the conference. Previous analyses have shown reports average a 4.1 rating but those evaluations were not done using standardized rubrics.

\section{An Understanding of Professional and Ethical Responsibility}

This year we have added evaluation of the "ethics" outcome from EC 2000. The evaluation question probes the handling of proprietary information, licensed software, confidentiality, conflicts of interest, citation of sources, quotations, and copyrights. It also evaluates the extent to which discussion of the impact of technology on society reflected recognition of the social responsibilities of scientists and engineers. The rubric is shown below, but data is not available as of this writing. Our international experience is not necessarily designed to address ethics issues but we will use this year's analysis to assess the degree to which this ability may be an indirect outcome of the student experience. 


\section{Rating 5: excellent}

\section{Professional and Ethical Responsibility Rubric}

The report clearly indicates and stresses the importance of the impacts of technology on society and our need to be concerned about them or explicitly acknowledges the responsibility borne by technologists for the effect of their professional work on society.

\section{Rating 3: acceptable}

The report clearly indicates the importance of the impacts of technology on society.

\section{Rating 1: poor}

The report indicates that the students believe that the impacts of technology on society are worth investigating but does not stress their importance.

\section{Summary and Future Directions}

This paper reviews only a few of the items that comprise our overall report evaluation. Hopefully they provide an illustration of our approach and some guidelines for evaluation questions and rubrics that others could adopt for their own purposes. These could be applicable for a variety of academic activities that address the outcomes described above.

As has been mentioned, we currently evaluate the major student product---the final report. Discussion is underway aimed at evaluating the process. This issue is problematic since the process is directly dependent upon advising methodologies that vary among faculty advisors. All of this is also somewhat political since many believe there is strong connection between high quality advising and highly rated reports. The issue gets interesting when the correlation between low quality reports and poor advising is discussed.

Our continuous improvement feedback loop is under development. The report issued by the coordinator of the summer review usually provides specific guidelines for improving overall IQP quality. However, an enforcement mechanism for implementation is informal and often nonexistent. Issues of academic freedom and recognition of good advising get confused with evaluation of student outcomes. We are actively pursuing effective solutions for these problems.

\section{Bibliography}

1. T.M. Davis, editor, Open Doors 1998/99: Report on International Educational Exchange, Institute of International Education, New York, N.Y. (http://www.opendoorsweb.org/)

2. Mello, N.A., How Can Universities Provide a Global Perspective for Engineers? One Institution's Solution, ASEE Annual Conference Proceedings, St. Louis, MO, June 2000.

3. D. DiBiasio, N. Mello, and D. Woods, "Multidisciplinary Teamwork: Academic Practices and Assessment of Student Outcomes”, Best Assessment Processes III Conference, Rose-Hulman University, April, 2000.

DAVID DIBIASIO is Associate Professor of Chemical Engineering and Assessment Coordinator for the Interdisciplinary and Global Studies Division at WPI. He received his $\mathrm{PhD}$ in chemical engineering from Purdue University and has worked for the DuPont Company. His educational work focuses on educational assessment, active and cooperative learning, writing and chemical engineering, and international engineering education. 